\title{
La gestión local del riesgo en el marco de las transformaciones sociales contemporáneas ${ }^{1}$
}

\section{Local risk management in the framework of contemporary social transformations}

\author{
Laura Cerdas Guntanis \\ Escuela de Trabajo Social, \\ Universidad de Costa Rica, Costa Rica \\ laura.cerdas@ucr.ac.cr \\ https://orcid.org/0000-0003-4894-9111
}

\section{Resumen}

Introducción: este artículo deriva del Trabajo Final de Graduación titulado Gestión del Riesgo ante desastres: fundamentos éticos y perspectivas teórico metodológicas, del Programa de Posgrado en Trabajo Social con énfasis en Investigación.

Objetivo: analizar en el desarrollo histórico del capitalismo las principales inflexiones teórico - metodológicas y la direccionalidad ético política, correspondiente con el origen y la consolidación del enfoque de la gestión local del riesgo.

Método: la investigación se desarrolló desde una perspectiva histórico crítica, haciendo uso de técnicas como la revisión bibliográfica y documental, así como entrevistas semiestructuradas a personas expertas, dada su experiencia institucional y de investigación en el tema.

Resultados: la reconstrucción de la historicidad de los fundamentos de la gestión local del riesgo en la dinámica del sistema capitalista, implicada en la doble relación: Naturaleza Sociedad e Historia - Teoría, representó el marco histórico - teórico para la discusión en torno al carácter social del riesgo, así como de la dimensión local de su gestión en el contexto de las transformaciones sociales contemporáneas.

Conclusiones: la génesis del riesgo que enfrentan las poblaciones en los espacios locales coloca el imperativo ético y político de visibilizar la desigualdad como base material de la vulnerabilidad. Implica, por tanto, el cuestionamiento en torno a las particularidades que adquiere la gestión del riesgo y sus finalidades, al plantearse como transversal en las políticas de desarrollo.

Palabras clave: Desastres, Desigualdad social, Participación, Desarrollo local, Trabajo Social.

\footnotetext{
${ }^{1}$ Este artículo surge de la ponencia presentada en las Jornadas de Investigación de la Escuela de Trabajo Social «Tendencias Contemporáneas de la investigación en Trabajo Social», realizadas en el mes de octubre del año 2021.
} 
DOI 10.15517/ rr.v101i2.49275

\begin{abstract}
Introduction: this article derives from the Final Graduation Project entitled Disaster Risk Management: ethical foundations and theoretical-methodological perspectives, as part of the Postgraduate Program in Social Work, with an emphasis on Research.

Objective: analyze in the historical development of capitalism the main theoreticalmethodological inflections and the ethical-political directionality, corresponding to the origin and consolidation of the local risk management approach, highlighting the social nature of risk, as well as the local dimension of its management.

Method: the research was developed from a critical historical perspective, making use of techniques such as bibliographic and documentary review, as well as semi-structured interviews with experts, given their research and institutional experience on the subject.

Results: the reconstruction of the historicity of the foundations of local risk management in the dynamics of the capitalist system, implied in the double relationship: Nature - Society and History - Theory, represented the historical - theoretical framework for the discussion around the social character of the risk, as well as the local dimension of its management in the context of contemporary social transformations.

Conclusions: the genesis of the risk that populations face in local spaces places the ethical and political imperative of making inequality visible as the material basis of vulnerability. It implies, therefore, the questioning around the particularities that risk management acquires and its purposes, when considering itself as a transversal in development policies.
\end{abstract}

Keywords: Disasters, Social inequality, Participation, Local development, Social work.

\title{
Introducción
}

La lectura analítica de los escenarios que estamos enfrentando en la contemporaneidad, colocándonos desde la lógica de los desastres, nos permite distinguir la materialización creciente de las expresiones concatenadas de la acumulación histórica del daño ambiental, así como de la desigualdad social y económica; mostrándonos que estamos frente a escenarios de riesgo ante desastres cuya causalidad es histórica.

Desde este marco, ha sido parte de los intereses de investigación de la autora profundizar en la discusión de la historicidad del riesgo, así como de sus repercusiones en el carácter diferencial de su impacto en las condiciones y medios de vida de las poblaciones, por tanto, en las posibilidades que poseen los actores sociales para su gestión, así como para la prevención y manejo de los desastres desde los espacios locales.

Así pues, el presente artículo se deriva del Trabajo Final de Graduación, realizado por la autora, titulado «Gestión del Riesgo ante desastres: fundamentos éticos y perspectivas teórico metodológicas», el cual se aprobó en el año 2016 como parte del Programa de Posgrado en Trabajo Social, con énfasis en Investigación. A partir de este estudio, se 
contribuyó a la discusión en torno a este enfoque, el cual ha sido parte de los procesos de investigación, docencia y acción social desarrollados por la autora en la Escuela de Trabajo Social de la Universidad de Costa Rica.

La investigación se planteó como objeto de estudio: la historicidad de los fundamentos teórico metodológicos y ético políticos predominantes del enfoque de la Gestión Local del Riesgo, en la complejidad de la dinámica del sistema capitalista, para lo cual se consideró pertinente fundamentarla desde una perspectiva histórico - crítica; lo anterior, en tanto la tendencia develada por el estado del arte, de reproducir y no debatir sobre los planteamientos conceptuales de este enfoque y su trayectoria histórica, así como de enfatizar en propuestas de orden metodológico en respuesta a escenarios de riesgo, fueron relevantes para reflexionar en torno a la importancia de discutir sobre la historicidad del enfoque de la Gestión del Riesgo.

Relacionar el objeto de estudio en su historicidad con la lógica del desarrollo del capitalismo coadyuvó a comprender el proceso de consolidación histórico - teórico de dicho enfoque, mediado por la configuración de las explicaciones dadas a los desastres en diferentes épocas históricas. Estas explicaciones han estado asociadas a procesos de orden económico, político y social dando lugar a comprensiones que fueron posicionándose de forma hegemónica para el estudio y abordaje del tema, conocidas como paradigma fiscalista y paradigma social del desastre.

El reconocimiento de aquellas principales continuidades, inflexiones y rupturas teórico metodológicas en la comprensión del desastre, así como también éticas y políticas de los intereses contenidos en la direccionalidad de las formas de abordaje, dan cuenta de la problematización que desde las ciencias sociales y posteriormente desde la interdisciplinariedad permitió trascender las concepciones fisicalistas, para comprenderlos como procesos sociales e históricos en estrecha relación con los modelos de desarrollo.

La categoría riesgo emerge en este contexto de debates y rupturas para explicar el origen, desarrollo e impacto de los desastres, desde una perspectiva social e histórica. Para ello, fue de gran importancia la comprensión de la vulnerabilidad a partir de la década de los años setenta, como categoría explicativa de la incidencia que tienen los modelos del desarrollo y las condiciones de vida de las poblaciones en la generación del riesgo y por tanto en la ocurrencia de los desastres.

Es así como, el riesgo, desde diversas comprensiones teóricas y epistemológicas, ha conllevado a la visibilización de las causas sociohistóricas, así como de las particularidades que adquieren las repercusiones de los desastres. Lo cual, ha incidido en posicionar formas de abordaje con finalidades distintas a las tradicionalmente centradas en los daños y las pérdidas, develando la importancia de gestionar de manera permanente el riesgo.

El enfoque de la Gestión del riesgo ante desastres surge y se consolida en América Latina, en el marco de estos debates, a partir de la década de los años noventa, colocando la importancia de gestionar localmente el riesgo con la participación de múltiples actores 
sociales y por tanto de transversalizar la identificación, el control y la reducción del riesgo como parte de la gestión del desarrollo humano y sostenible.

Desde entonces, dicho enfoque se ha posicionado en la agenda de investigación científica, así como en los marcos políticos y legales que en esta materia rigen el accionar institucional a nivel mundial y en nuestro país.

La reconstrucción de la historicidad de los fundamentos de la Gestión local del riesgo en la dinámica del sistema capitalista, implicada en la doble relación: Naturaleza - Sociedad e Historia - Teoría, conllevó a discutir la historicidad del riesgo y sus procesos de gestión local en el contex to de las transformaciones sociales, tema en el que particularmente se centra el presente artículo. Esto con la finalidad de aportar, desde la investigación realizada, al debate interdisciplinario en torno al carácter social e histórico del riesgo y las posibilidades e implicaciones de transversalizar su reducción en la gestión del desarrollo.

\section{El imperativo de tornar la mirada a las causas: la historicidad del riesgo y de los desastres}

Los debates y rupturas con el carácter fisicalista de los desastres, desde el paradigma social, dan cuenta del posicionamiento de la vulnerabilidad como categoría explicativa de la incidencia que tienen las condiciones de vida, además de los fenómenos naturales, en la construcción del riesgo y por ende en la ocurrencia del desastre. Esto no solo transformó la concepción del desastre, sino también la direccionalidad de sus formas de investigación y de abordaje, donde las «causas», además del «impacto», adquirieron relevancia.

El análisis de la vulnerabilidad de las poblaciones, en esta línea, resulta fundamental en la comprensión del riesgo que enfrentan, pues así se pone en debate aquellos procesos económicos, sociales y políticos que asociados a patrones de desarrollo repercuten en la desigualdad entre la población (Maskrey 1989, Hewitt 1996, Blaikie et al. 1996). Desde esta perspectiva y coincidiendo con los aportes de tales autores y autoras, la vulnerabilidad trasciende la exposición a las multiamenazas, es decir ser «vulnerable ante la posibilidad de ocurrencia de ciertos fenómenos naturales» para en su lugar evidenciar aquellos procesos que derivados del orden económico y social capitalista deterioran las condiciones y medios de vida de las poblaciones, lo cual media no solamente en la construcción del riesgo, sino también en el carácter diferencial del impacto del desastre y, por ende, de las posibilidades que tanto la población como la institucionalidad poseen para enfrentarlo.

Los aportes que esta lectura de la vulnerabilidad brinda a la comprensión del carácter social del riesgo y por ende del desastre ha derivado en tendencias de análisis que discuten acerca del riesgo cotidiano, el riesgo estructural, o bien, más recientemente, de los factores subyacentes del riesgo, así planteado en los marcos políticos y legales a nivel internacional y nacional.

Desde estas tendencias se hace referencia a aquellas condiciones de riesgo existentes en la sociedad y que han sido derivadas de los procesos de transformación social, económica 
y política, condiciones que a su vez pueden agudizarse por la ocurrencia de un desastre. Tal como Maskrey (1993) hace referencia, el desastre resulta de la aceleración que una amenaza natural puede generar al desastre cotidiano que diversas poblaciones enfrentan a partir de sus condiciones de vida.

A partir de estas reflexiones, desde las cuales se han tejido vínculos entre el desarrollo y la vulnerabilidad, así como más recientemente entre el riesgo y el desarrollo, quisiera detenerme, en tanto interpela a la aprehensión de la génesis del riesgo, su relación con las condiciones y medios de vida, así como las finalidades que asumen los procesos de gestión del riesgo, entre estos aquellos que se impulsan desde lo local.

Un punto de partida que nos acerca a la aprehensión de la complejidad del riesgo, obedece a una lectura histórico crítica que sitúa su origen y desarrollo en la dialéctica relación ser humano - naturaleza, donde esta última se constituye en objeto de transformación para la satisfacción material de las necesidades humanas. La perspectiva ontológica de la relación ser humano - naturaleza permite comprender la direccionalidad que asumen las relaciones que históricamente se han establecido desde la humanidad con la naturaleza, su expoliación mediante procesos productivos cada vez más extractivos, que coloca a los seres humanos en formas diversas de relación con ésta; relaciones sentadas, por sí, en condiciones de desigualdad, que median la génesis y desarrollo del riesgo ante desastres.

Desde esta perspectiva, partimos de que históricamente la transformación de materias primas naturales ha sido fundamental para la producción y reproducción de las condiciones materiales de existencia de los seres humanos, de manera que «la sociedad no puede existir sin la naturaleza - después de todo, es la naturaleza, transformada por el trabajo, que proporciona las condiciones de mantenimiento de la vida de los miembros de la sociedad. Cualquier sociedad humana tiene su existencia hipotecada a la existencia de la naturaleza - lo que varía históricamente es la forma de la relación de la sociedad con la naturaleza: varían, a lo largo de la historia, el tipo de transformación que, a través del trabajo, la sociedad opera en los elementos naturales para que puedan servir, y los medios empleados en esta transformación (Netto y Braz 2007, 35) » $^{2}$

Por tanto, las formas y los medios empleados para la transformación de la naturaleza a través del trabajo, como fundamento ontológico de la sociedad, condición de vida del ser humano, perenne e inherente a la vida humana (Luckács 2004), varían históricamente, es decir, la relación de la sociedad con la naturaleza adquiere particularidades y rumbos distintos. Esto remite a reflexionar en torno a las relaciones sociales que median la

\footnotetext{
${ }^{2}$ A sociedade não pode existir sem a natureza - afinal, é a natureza, transformada pelo trabalho, que fornece as condições de sustentação da vida para os membros da sociedade. Toda e qualquer sociedade humana tem a sua existência hipotecada à existência da natureza - o que varia historicamente é a modalidade de relação da sociedade com a natureza: variam, ao londo da história, os tipos de transformação que, através do trabalho, a sociedade opera nos os elementos naturais para deles se servir, bem como os meios empregados nessa transformação (Netto y Braz 2007, 35).
} 
producción y reproducción de las condiciones materiales de existencia de las y los sujetos, por tanto, contemplar los diversos modos de producción, las consecuentes formas de organización que han generado en la sociedad y sus implicaciones en la transformación del ambiente, así como en las condiciones y medios de vida de las poblaciones.

Desde el modo de producción capitalista, el carácter de la relación sociedad naturaleza fue tornándose progresivo, cada vez más extractivo e intensivo, en virtud de la potenciación del desarrollo de las fuerzas productivas y de los intereses de acumulación del capital. El desarrollo de la producción industrial daba cuenta desde las fases iniciales del capitalismo de las marcas en la explotación de los recursos naturales inherentes al proceso histórico de consolidación y expansión como orden económico mundial. Lo anterior, determinado en ese entonces por la explotación y uso de materias primas, los procesos de urbanización y ocupación del territorio, así como del desarrollo científico y técnico al servicio de la producción, el cual fue alcanzado niveles mayores de evolución, con repercusiones en la invención de métodos a favor de la productividad, es decir, en la dominación de la naturaleza.

En este contexto, donde la naturaleza se ha constituido en objeto de explotación, tanto en forma intensiva, como extensiva en razón del desarrollo de la producción y la acumulación capitalista, es posible palpar cómo las implicaciones en el deterioro y destrucción de los recursos naturales son cada vez mayores, amenazando la propia conservación de la naturaleza y de la vida humana.

Al respecto el Informe de Evaluación Global sobre la Reducción del Riesgo de Desastres emitido por parte de la Oficina de las Naciones Unidas para la Reducción del Riesgo de Desastres UNIDSR (2015) plantea que, «el consumo excesivo de energía, agua y otros recursos como resultado del crecimiento económico ha superado la biocapacidad del planeta, traspasando los límites de los sistemas planetarios cruciales y amenazando la supervivencia humana. Muchos ecosistemas que proporcionan servicios de protección y suministro esenciales se están degradando más allá del punto de recuperación, mientras los cambios en las temperaturas, las precipitaciones, el nivel del mar y otros factores resultantes del cambio climático global modifican los patrones de las amenazas y hacen que aumente el riesgo de desastres (xvi)».

En lo anterior, se devela que de mantenerse las tendencias de crecimiento económico y de consumo «para el año 2030 se habrá superado la biocapacidad del planeta en aproximadamente un $200 \%$. En otras palabras, se necesitarán dos planetas adicionales para sostener el consumo y absorber los deshechos»(UNIDSR 2015, 269). Así, el cambio climático, la reducción de la biodiversidad y la mermada disponibilidad del agua conducirán a un aumento del riesgo de desastres, en términos de sus repercusiones en la alteración de los patrones de las amenazas naturales de origen meteorológico, en relación con su frecuencia e intensidad (UNIDSR 2015). A esto se suman las repercusiones en la reproducción de las 
condiciones y medios de vida de las poblaciones, si tornamos la mirada a los efectos en las actividades productivas, así como en la seguridad alimentaria.

Por tanto, las contradicciones existentes entre las tendencias aceleradas de consumo de los recursos y las capacidades de la naturaleza para restituirlos, en el caso de aquellos recursos que aún se consideran renovables, han ampliado el debate en torno a las implicaciones de los modelos de desarrollo en la degradación del ambiente, en la seguridad de la vida humana y, por ende, en la generación del riesgo que enfrentan las poblaciones ante los desastres. Más aún cuando tales contradicciones se expresan desigualmente en el planeta, acorde con la ubicación geográfica, la economía y las posibilidades de desarrollo de cada país.

Este debate en torno a la relación desarrollo - ambiente - riesgo, se problematiza en un contexto en el cual, siguiendo a Dierckxsens «el cuido de la vida humana y natural resulta incompatible con la lógica del capital» $(2006,37)$, lógica desde la cual la destrucción de la riqueza natural se transforma en producción de riqueza material, provocando desequilibrios naturales cada vez mayores. Es aquí donde, refiere el autor, se desarrolla la resistencia que reivindica la conservación de la naturaleza como patrimonio común de la humanidad, en la que la pérdida de la vida natural tanto para las generaciones actuales como futuras no se contabiliza en una economía de mercado.

De esta forma, las propuestas de desarrollo sostenible que fueron adquiriendo relevancia en la agenda mundial ambiental, desde la década de los años setenta y con más fuerza desde los noventa, se tornan contradictorias en términos de sus finalidades, cuando estas pretenden impulsarse desde la lógica capitalista cuya sostenibilidad, según Dierckxsens (2006), depende más bien del sacrificio perpetuo de la vida natural y humana.

Molina Molina (2007), en esta línea, agrega cómo la contradicción entre el proceso de reproducción ampliada del capital y la incapacidad de la naturaleza para regenerar al mismo ritmo sus recursos obliga al capital a la conservación de la misma, así como a intervenir en su proceso de regeneración dando lugar al denominado «desarrollo sostenible». Esto, como parte de la reflexión que se ha venido planteando, conlleva a cuestionarse ¿desarrollo sostenible para quiénes?, pues en estos términos la autora asegura que obedece a un desarrollo sostenible para el capital.

De manera que podríamos afirmar cómo en nombre de la conservación de la naturaleza para las generaciones actuales y futuras se garantiza la perpetuación de este orden económico mundial, confrontándonos a una encrucijada ética, más aún cuando el «desarrollo sostenible» sigue proponiéndose como parte de las finalidades de los procesos para la gestión local del riesgo ante desastres desde los marcos políticos - legales de nuestro país y a nivel mundial.

El análisis realizado hasta el momento permite evidenciar cómo inherente a la producción y reproducción de la riqueza material se acumula el daño ambiental, lo cual, concatenado a la creciente desigualdad social y económica propia de este orden capitalista, 
configura, a mi modo de ver, un espectro para aprehender la historicidad de la génesis y desarrollo del riesgo ante los desastres.

Netto y Braz (2007) nos recuerdan que en el contexto de las grandes transformaciones que ha experimentado el desarrollo del capitalismo a lo largo de la historia, un proceso constitutivo a la acumulación del capital obedece a una riqueza que exponencialmente se expande ante la desigualdad que enfrentan masas de hombres y mujeres cuyo acceso a los bienes necesarios de vida son extremadamente limitados.

Esta contradicción genética del capitalismo, derivada de una producción cada vez más socializada en relación a una apropiación privada de la riqueza (lo cual da contenido a la contradicción entre el capital y el trabajo), remite a considerar las expresiones de desigualdad económica, social, política y cultural, producto de esta contradicción, comprendidas como las manifestaciones de la cuestión social, las cuales históricamente han incidido en el deterioro de las condiciones y medios de vida de las poblaciones; segunda de las aristas desde las cuales es posible comprender la historicidad del riesgo.

Un resultado ha sido la radicalización de la concentración de los ingresos, de la propiedad y del poder, en contrapartida con un violento empobrecimiento de la población, una ampliación brutal del desempleo y el subempleo, el desmontaje de los derechos conquistados y de las políticas sociales universales, imponiendo un sacrificio forzado a toda la sociedad. La reestructuración de la producción y los mercados apoyados más en métodos de consumo intensivo de la fuerza de trabajo que en innovaciones científicas y tecnológicas de última generación, se suman retrocesos en la relación entre el Estado y la sociedad cuando la referencia es la vida de todos y la protección de los derechos conquistados por la mayoría (Iamamoto Villela 2004, 20) ${ }^{3}$.

El proyecto neoliberal que rige los modelos de desarrollo de nuestros países, mediado por los procesos de transformación del Estado, nos coloca en un contexto donde las posibilidades de reproducción de las condiciones de vida se limitan a la venta de la fuerza de trabajo en condiciones cada vez más alienantes y restrictivas de derechos.

A su vez, las políticas sociales lejos de asumir la cuestión social en su integralidad, tomando en cuenta sus múltiples y diferenciadas expresiones, pero más aun considerando los procesos sociales contradictorios que las originan y las transforman, fragmentan la cuestión social en diversos «problemas sociales». Siguiendo a Iamamoto (2004), esos problemas sociales se consideran desde una óptica que los analiza y aborda como problemas del individuo y de la familia despojándoles de una dimensión colectiva y eximiendo a la sociedad

\footnotetext{
${ }^{3} \mathrm{O}$ resultado tem sido uma ampla radicalização da concentração de renda, da propriedade e do poder, na contrapartida de um violento empobrecimento da população, uma ampliacão brutal do desemprego e do subemprego, o desmonte dos direitos conquistados e das políticas sociais universais, impondo um sacrifício forçado em toda a sociedade. A reestruturação da produção e dos mercados, apoiada mais em métodos de consumo intensivo da força de trabalho do que em inovações científicas e tecnológicas de última geração, somam - se mudancas regressivas na relação entre Estado e sociedade quando a referência é a vida de todos e os direitos conquistados pelas grandes maiorias (Iamamoto Villela 2004, 20).
} 
de clases de la responsabilidad en la producción de las desigualdades sociales, contribuyendo a su naturalización.

Es en este contexto de relaciones sociales históricamente asentadas en la desigualdad que se reproducen relaciones diferenciales con la naturaleza, unas para la acumulación de riqueza y otras por necesidades de sobrevivencia, las cuales a su vez son expoliadoras del ambiente, aunque en condiciones materiales y direccionalidades ético políticas notoriamente diferentes. Es así como encontramos poblaciones ubicadas en territorios no aptos para los asentamientos humanos o para el desarrollo de sus actividades productivas, en tierras que suelen ser sus únicos medios de subsistencia.

Las condiciones de desigualdad que se generan del tipo de organización de la sociedad actual, por tanto, además de incidir en la configuración del riesgo, impiden que haya condiciones óptimas para que tanto las poblaciones como la institucionalidad inserta en esta lógica puedan enfrentarlo. Desde esta lectura, podemos visualizar cómo las manifestaciones de la cuestión social inciden en la conformación del riesgo, las cuales desde una relación dialéctica se agudizan y recrudecen con la ocurrencia de los desastres.

De manera que colocar la comprensión del origen y desarrollo del riesgo en esta lectura desafía a mirar los escenarios de riesgo desde la totalidad social, la cual se reproduce en las particularidades de los espacios locales y en la singularidad de las familias. El origen e impacto de los desastres, por tanto, tiene una historicidad que implica dialécticamente la relación naturaleza - sociedad y en particular naturaleza - sistema económico y social y sus particulares procesos de producción y reproducción social. A este rasgo le complementa la creciente y polarizante desigualdad social en la distribución de la riqueza, así como en los medios de vida y de trabajo.

Esto exige desmontar las causas históricas de esos escenarios de riesgo a nivel local, que facilite más allá de identificar aquellas condiciones que hacen susceptibles a las poblaciones frente a las amenazas naturales, usualmente identificadas como condiciones de vulnerabilidad, a tornar la mirada al conjunto de las desigualdades económicas, sociales, políticas y culturales que se reproducen en la vida cotidiana de las poblaciones, las cuales de por sí las colocan en desigualdad en el acceso a sus derechos, incidiendo así en el detrimento de sus condiciones y medios de vida.

Cabe cuestionarse, en este sentido, en torno a si estamos frente a ¿poblaciones vulnerables o bien vulnerabilizadas? Más allá del riesgo y del desastre, estamos frente a poblaciones cuyas condiciones de existencia se tornan cada vez más vulnerables. Por tanto, si bien el riesgo se constituye en nuestro ángulo de visión para acercarnos a las localidades, compete problematizar con los actores locales el origen de sus condiciones de vida, agudizadas por el riesgo y recreadas por los desastres, lo que imprime una direccionalidad distinta a los procesos de gestión. 
DOI 10.15517/ rr.v101i2.49275

\section{Los procesos de gestión local del riesgo y sus finalidades, en el marco de las transformaciones sociales}

La discusión desarrollada con antelación, permite evidenciar que en definitiva no estamos ante eventos o fenómenos naturales aislados cuando de desastres se trata, sino ante procesos históricos de construcción del riesgo, cuya génesis se articula con las transformaciones sociales, económicas y políticas inherentes a las tendencias que rigen los modelos de desarrollo de nuestros países.

Esta lectura del carácter social de los desastres y del riesgo dio lugar al planteamiento de propuestas de abordaje que sobrepasan los límites de la atención inmediata de los daños y las pérdidas post desastre, para posicionar la importancia de gestionar el riesgo. De esta manera, el enfoque de la gestión del riesgo representó una ruptura no solamente en la comprensión del desastre, sino también en las formas y finalidades de su abordaje, donde adquirían relevancia la reducción del riesgo, la participación de los actores sociales, así como las posibilidades de desarrollo de las localidades.

La gestión local del riesgo, acorde con las propuestas de las personas e ideólogas de la Red de Estudios Sociales en Prevención de Desastres en América Latina (LA RED), constituye un proceso que se desarrolla desde los espacios locales con la participación de multiplicidad de actores sociales, en la construcción de aquellas alternativas que, en respuesta a la identificación, control, reducción y previsión del riesgo, apuesten por el desarrollo sostenible

Dada la importancia que adquiere gestionar el riesgo desde las localidades, cabe detenerse en la reflexión en torno a tres aspectos que remiten a discutir las particularidades que asumen estos procesos en la contemporaneidad.

\section{La comprensión de lo local}

Lo local remite, en primera instancia, al territorio en el cual se reproduce y materializa el riesgo, así como donde son perceptibles los daños y las pérdidas por desastres, particularmente desde la percepción de los actores sociales, a raíz del impacto en su vida cotidiana; representa el espacio, por tanto, para emprender aquellas acciones orientadas a la reducción del riesgo y el manejo de los desastres.

Tomar como punto de partida el territorio implica considerar que este podría trascender las divisiones político administrativas - llámese provincias, cantones y distritos en tanto comprende el riesgo como resultado de múltiples procesos concatenados, cuyos orígenes trascienden lo local; esto conlleva una articulación con otros niveles territoriales para la identificación y gestión de los procesos causales del riesgo (Centro de Coordinación para la Prevención de los Desastres Naturales en América Central CEPREDENAC Programa de las Naciones Unidas para el Desarrollo PNUD 2005).

De ahí que, acorde con estos autores, se plantea la importancia de no supeditar lo local al municipio, relación comúnmente establecida que podría incidir en homologar la 
gestión local a una gestión municipal del riesgo, pues en función de los procesos causales del riesgo, es posible impulsar proyectos intermunicipales, por criterio de cuenca hidrográfica, o bien, en territorios como son los distritos y sus poblados.

Lo local desafía entonces su delimitación a partir de límites geográficos y político administrativos para reconocer que aquellos territorios en los cuales se materializa el riesgo y los desastres se encuentran insertos en la totalidad social y reproducen las particularidades de los países; de manera que no se explican solamente por sus características geográficas, sociodemográficas o bien geomorfológicas, sino como resultado de procesos históricos, culturales, económicos, políticos y sociales, articulados con la legalidad social contemporánea. Desde esta comprensión, los espacios locales, siguiendo a Molina Molina (2006, 129), pueden estar demarcados por identidades históricas, culturales y delimitaciones convencionales de límites geográficos, no obstante, es importante reconocer que en estos subyace y se reproduce «la compleja trama de relaciones diferenciales en razón de: la posición social y económica, así como, el ejercicio del poder y la comprensión de diversidad socioeconómica, cultural, de género y edad».

Así, es en el espacio local donde las manifestaciones de la cuestión social, asentadas en la desigualdad, se reproducen en la cotidianidad de los sujetos y sus familias que se enfrentan día a día a la precarización de sus condiciones de trabajo y del desarrollo de sus actividades productivas, al desempleo, la pobreza, así como al no acceso a los derechos de atención óptima de la salud, la educación, vivienda digna, agua potable, saneamiento ambiental, entre otros, que tornan vulnerables la reproducción de sus condiciones materiales de existencia.

Partir de esta comprensión teórica y metodológica desafía, como parte de los procesos en esta materia, a incorporar la investigación en torno a las condiciones y medios de vida de las poblaciones, develando las manifestaciones de la cuestión social que han mediado en su deterioro y a la vez han constituido aquellas demandas locales, entre las cuales puede estar el riesgo. De manera que sea posible reconstruir junto con los actores locales los procesos que históricamente han configurado su espacio local, el detrimento de sus condiciones de vida, la explotación de los recursos naturales y por tanto el riesgo que enfrentan ante los desastres.

Aunado a la identificación de las multiamenazas, esta lectura coadyuva a problematizar el carácter histórico, dinámico y particular del riesgo que enfrentan las poblaciones de una misma localidad, o bien, del riesgo que se materializa en cada uno de los poblados de un mismo distrito, por ejemplo. Lo anterior, desafía a la identificación de los escenarios de riesgo que se realiza desde una comprensión endógena, homogénea y hasta aparente (desarticulada de la totalidad social que invisibiliza las diferencias de clase), para avanzar hacia la problematización de las causas de las expresiones del riesgo que enfrentan cada día las poblaciones e inciden en el deterioro de sus condiciones de vida, ya por sí agudizadas por el sistema. 


\section{Rasgos que adquieren los procesos de gestión local del riesgo}

Los planteamientos del enfoque de la gestión local del riesgo resaltan, desde sus orígenes, el carácter participativo e inclusivo de una serie de actores sociales, entre estas organizaciones locales, instituciones del Estado y empresas privadas en la identificación de los escenarios de riesgo, así como en la gestión de estrategias que contribuyan a su reducción. Así pues, la gestión local obedece a un proceso «cuyo impulso, concreción y apropiación son locales, logrados a través de los actores locales, quienes se relacionan o se identifican con el nivel local en lo que se refiere a su quehacer diario, productivo o familiar y tienen sentido de pertenencia a esos niveles» (CEPREDENAC - PNUD 2005, 43).

La participación se constituye, por tanto, en un rasgo inherente a la gestión local «un mecanismo de legitimación y de garantía de pertenencia, y la piedra angular de la apropiación del proceso por parte de los actores locales» (CEPREDENAC-PNUD 2005, 43). De esta forma, se apuesta a que la gestión local se encuentre mediada por el desarrollo de procesos participativos que favorezcan el reconocimiento del riesgo desde la percepción de los actores locales, así como una gestión que responda a sus demandas, intereses y expectativas de desarrollo local, clave para la apropiación y sostenibilidad de los procesos.

Es en este sentido que se plantea la importancia de conformar estructuras sociedad civil - institucionalidad que permitan articular la gestión local del riesgo en determinado territorio con otros niveles territoriales, por ende, con la diversidad de actores sociales. Asimismo, estructuras que coadyuven a impulsar y coordinar la articulación entre la gestión del riesgo y la gestión del desarrollo local, en aras de que las acciones a nivel estatal y local se orienten a incorporar aquellas prácticas necesarias para reducir el riesgo existente y controlar la gestación de condiciones de riesgo con una perspectiva de sostenibilidad e integralidad (CEPREDENAC-PNUD, 2005).

Congruente con estos planteamientos, el marco político legal costarricense en esta materia plantea la transversalización de la gestión del riesgo en la labor del Estado costarricense, instaurándose espacios de coordinación entre diversos actores para impulsar la reducción del riesgo como parte de las políticas de desarrollo nacional y local. Lo anterior, desafía a impulsar aquellas estrategias que desde las particularidades de los espacios locales apunten a fortalecer la participación con incidencia política por parte de los actores sociales, que les permita ejercer un papel activo en la construcción, negociación y seguimiento de agendas de gestión entre organizaciones locales e instituciones con presencia física y administrativa en tales espacios.

El reconocimiento del riesgo y la toma de decisiones sobre su reducción deben traducirse entonces en estrategias planteadas para la exigibilidad de las demandas locales, confrontadas con los derechos económicos, sociales y ambientales no realizados. Esto implica la problematización junto a los actores de aquellas manifestaciones de la cuestión 
social que han incidido históricamente en el deterioro de sus condiciones de vida, por lo tanto, en la génesis y materialización del riesgo.

Lo anterior coloca como parte de la direccionalidad ética y política de la gestión del riesgo fortalecer los procesos que desde lo local permitan evidenciar las respuestas y omisiones de las políticas estatales y su incidencia en la generación del riesgo, así como gestionar ante las instituciones y las empresas privadas el cumplimiento de las responsabilidades que a nivel político legal les competen en materia de reducción del riesgo, con una clara intencionalidad de reconocer y dar respuesta a las demandas locales, desde la protección, defensa y exigibilidad de los derechos económicos, sociales y ambientales. Dicha direccionalidad incide en el fortalecimiento de una autonomía crítica en la participación de la gestión del desarrollo de su localidad, entendida acorde con Pereira Pereira $(2002,87)$ «a la capacidad de las personas de no apenas saber elegir y evaluar informaciones con vistas a la acción, sino de criticar y si es necesario, cambiar las reglas y las prácticas de la cultura a la que pertenecen».

Desde esta intencionalidad no caben procesos de gestión del riesgo impulsados, en la mayoría de las ocasiones, por actores sociales externos en los espacios locales, con intereses diversos desde los cuales la participación no trasciende el carácter consultivo y enfocado al manejo del desastre como tal. Tampoco aquellos procesos orientados a la autogestión comunitaria del riesgo con enfoques resilientes, para que mediante la autoayuda y el voluntariado se asuman localmente las respuestas para enfrentar escenarios de riesgo cuyo origen es estructural.

Tales procesos carecen de espacios para la toma de decisiones en torno a las prioridades locales y las formas en las cuales interesa a las poblaciones gestionarlas, así como promueven estructuras organizativas que, en el contexto de las tendencias de desarrollo neoliberal, adquieren sentido desde una lógica del traslado de competencias tanto a las organizaciones locales, así como a las instituciones locales como las municipalidades, sin recursos financieros, técnicos, humanos para la ejecución de sus competencias.

La transversalización de la gestión local del riesgo en la gestión del desarrollo, implica, por tanto, que las poblaciones sean partícipes de procesos formativos en torno a las multiamenazas que enfrentan sus espacios locales, así como las causas del deterioro de sus condiciones y medios de vida, que conlleven a problematizar su incidencia en la generación de los escenarios de riesgo y su materialización en los desastres.

Asimismo, conlleva la construcción de agendas de proyectos desde lo local con contrapartes institucionales, que faciliten abordar las múltiples aristas de los escenarios de riesgo, en las plataformas intersectoriales donde la participación de las organizaciones locales y las poblaciones trascienda los comités para la atención de la emergencia a espacios de toma de decisiones colectivas desde expresiones organizativas autónomas, como podrían ser las redes locales. Plataformas en las que, de acuerdo con nuestro marco político legal, cada institución deberá transversalizar la gestión del riesgo en su quehacer, es decir, como parte 
de sus actividades ordinarias, lo cual representa un desafío tanto para la institucionalidad como para la ciudadanía en términos de la fiscalización del cumplimiento de esta responsabilidad.

Por otra parte, en términos del desastre como tal, la prevención, mitigación y manejo del desastre, desde la perspectiva de la gestión del riesgo se desarrollan como procesos, no como acciones aisladas enmarcadas en el antes, durante y después del desastre. Participar en la atención de los efectos del desastre resulta en sí un desafío en términos de desarrollar acciones en el marco de una estrategia de gestión estatal e institucional que, tal como se ha mencionado, en la mayoría de los casos es reactivo a la situación, debido a la toma de decisiones a nivel vertical hacia los espacios locales, esto con la finalidad de dar respuesta, rehabilitar y reconstruir las comunidades afectadas.

A ello se suman las implicaciones que los desastres tienen en la agudización del deterioro de las condiciones de vida de las poblaciones y las demandas que ello plantea a la institucionalidad, desde políticas sociales cada vez más focalizadas y restrictivas producto de los modelos de desarrollo económico neoliberal. Demandas que trascienden la inmediatez de la emergencia y, por ende, la asistencia social post desastre que se pueda desarrollar en torno a ello, de manera que las personas afectadas si no están siendo parte de los programas sociales de asistencia, eventualmente pasan a ser parte de estos de manera permanente.

Es posible entonces visualizar cómo desde la gestión del riesgo se coloca una direccionalidad distinta a la atención del estado de emergencia y a la reconstrucción de los espacios locales, desafiando la inmediatez a partir del desarrollo de acciones que impliquen un trabajo de recuperación del tejido social y de los intereses en conflicto; que contribuya además al análisis del escenario de riesgo recreado por el desastre en conjunto con los actores locales, así como al planteamiento de aquellas demandas que sean base para la toma de decisiones en los procesos de rehabilitación y recuperación en coordinación con las instituciones públicas y el sector privado.

Esto implica fuertes acciones de defensa - exigibilidad en el cumplimiento, manejo estratégico político de articulación de intereses y de confrontación con oponentes. Así, en la medida que se gestione localmente el riesgo, se gestionará localmente el manejo del desastre, con el objetivo de construir procesos tendientes a garantizar la satisfacción de aquellas demandas post desastre, desde el cumplimiento de la responsabilidad estatal en su función de protección social.

\section{Las finalidades de la gestión local del riesgo, a la luz de la aprehensión del riesgo como proceso social e histórico}

La discusión realizada hasta este momento permite visualizar cómo las finalidades de los procesos pueden estar orientadas al desastre, al riesgo, o al mejoramiento de las condiciones y medios de vida de las poblaciones. 
Hacia dónde tornar la mirada, parte de comprender que el abordaje de los desastres va más allá de estar preparados para atender las emergencias, trasciende entonces a la reducción del riesgo en la planificación del desarrollo en sus niveles nacional, regional y local. Es así como, desde esta finalidad, se plantea que «la gestión del desarrollo no puede ser vista sin referencia a la problemática del riesgo de desastre, y la gestión del riesgo no tiene mayor sentido si no se le ve como una dimensión de la gestión del desarrollo y como una práctica transformadora que apoya la búsqueda de mayores niveles de seguridad humana integral. La dimensión del desarrollo que debe caracterizar la intervención sobre el riesgo tal vez constituye su rasgo más esencial y definitorio» (CEPREDENAC-PNUD 2005, 48).

En esta línea, se pretende incorporar la reducción del riesgo en el quehacer institucional, así como de las organizaciones locales y empresas privadas, que facilite emprender una gestión correctiva y, más aún, prospectiva del riesgo en los procesos de transformación social y económica. La intención, por tanto, aduce a cómo desde la planificación del desarrollo se controla y reduce el riesgo y, en consecuencia, se logran pautas de desarrollo económico, ambiental y territorial sostenibles.

Tal como se ha venido explicitando, tornar la mirada a las condiciones de desigualdad social de las poblaciones que confluyen en la génesis y desarrollo del riesgo, más allá de contribuir a su reducción, prevención y eventual manejo del desastre, inscribe la gestión local en una lógica cuya intención trasciende a la exigibilidad de desarrollo y de realización de derechos económicos, sociales y ambientales de las poblaciones.

Lo anterior en búsqueda de que las localidades se consoliden en el medio en el cual se generen iniciativas y propuestas a ser articuladas - confrontadas con el Estado. Esta lectura desafía a repensar los espacios y posibilidades, planteadas desde el marco político legal costarricense, para la participación de las organizaciones locales, en sí de las poblaciones en la construcción de las estrategias y finalidades de los procesos de reducción del riesgo, más allá de la atención de las emergencias.

Asimismo, convoca a reflexionar en torno a las finalidades propuestas desde los marcos políticos legales a nivel mundial, reproducidos en aquellos que rigen las acciones en nuestro país, sobre la necesidad de «aumentar la resiliencia de las poblaciones frente al riesgo y los desastres». Lo cual, devela la importancia de discutir la direccionalidad que asumen las acciones en materia de gestión, las cuales más allá de un carácter transformador, parecieran naturalizar las condiciones de desigualdad, de riesgo y por ende la asistencia que en función de los mínimos vitales se brindan a estas poblaciones.

El debate por tanto queda abierto, en términos de las posibilidades y desafíos de emprender y fortalecer una gestión que partiendo de lo que pareciera urgente, como lo es el riesgo, o bien los escenarios de riesgo recreados por los desastres, se tejan procesos de fortalecimiento de las capacidades locales, en tanto actores locales artífices de agendas de desarrollo, como resistencia a las implicaciones de los modelos de desarrollo de corte neoliberal en sus condiciones y medios de vida. 
DOI 10.15517/ rr.v101i2.49275

\section{Conclusiones}

La aprehensión sociohistórica del objeto permite concluir que las diversas comprensiones dadas a los desastres se encuentran determinadas por los procesos económicos, sociales y políticos de los períodos históricos en las cuales emergen, de manera que como expresión de la relación historia - teoría, es desde la totalidad social y sus particularidades históricas que es posible aprehender la génesis y desarrollo de los paradigmas explicativos de los desastres: fisicalista y social.

La lectura de aquellas principales inflexiones y rupturas teórico metodológicas en la comprensión del desastre devela cómo en la génesis de la ruptura con el paradigma fisicalista del desastre la vulnerabilidad se constituyó a partir de la década de los setenta en la categoría explicativa de la incidencia que tienen las condiciones de vida de la población en la ocurrencia de los desastres, posicionando los aspectos sociales, económicos y políticos en la investigación del tema. Articulada a esta lectura, el riesgo se consolida como categoría explicativa del origen, desarrollo e impacto de los desastres en las condiciones y medios de vida de las poblaciones.

En este sentido, el enfoque de la gestión local del riesgo representa una síntesis de procesos y aportes conceptuales que se fueron problematizando en torno a la relación desastres - desarrollo, así como de la vulnerabilidad, el riesgo y los desastres como procesos sociales producto de la materialización del riesgo no manejado. Estos son fundamentos desde los cuales las formas de abordaje centran su atención en el conocimiento y atención de las causas, particularmente de las condiciones que vulnerabilizan las condiciones de vida con la participación de diversos actores sociales.

Posicionarse desde este enfoque interpela a emprender procesos analíticos del origen del riesgo que enfrentan las poblaciones en los espacios locales, en el contexto de los procesos de transformación social y económica de nuestros países, desde los cuales se plantea el imperativo ético y político de visibilizar la desigualdad, como base material de la vulnerabilidad. Por tanto, implica colocar la dimensión local de los procesos de gestión del riesgo en el marco de las tendencias globales, las transformaciones del Estado y las implicaciones éticas y políticas que podrían mediar la reducción del riesgo desde una perspectiva de desarrollo.

\section{Referencias}

Blaikie, Piers, Terry Cannon, Ian Davis, y Ben Wisner. 1996. Vulnerabilidad: el Entorno Social, Político y Económico de los Desastres. LA RED/ITDG. Bogotá, Colombia: Tercer Mundo.

CEPREDENAC (Centro de Coordinación para la Prevención de los Desastres Naturales en América y Central) PNUD (Programa de las Naciones Unidas para el Desarrollo). 
2005. La Gestión Local del riesgo. Concepto y Prácticas. Ecuador: Noción Imprenta Impresfergue.

Dierckxsens, Wim. 2006. «Utopía y sujeto en una economía alternativa». http://bibliotecavirtual.clacso.org.ar/Costa_Rica/dei/20120712025720/utopia.pdf

Hewitt, Kenneth. 1983. The Idea of Calamity in a Technocratic Age. London: Allen and Unwin. Edición en PDF.

1996. «Daños ocultos y riesgos encubiertos: Haciendo visible el espacio social de los desastres». En Desastres: Modelo para Armar. Colección de Piezas de un Rompecabezas Social, editado por Elizabeth Mansilla Menéndez, 11-29. Lima, Perú: LA RED

Iamamoto, Marilda. 2004. «Las dimensiones ético políticas y teórico metodológicas del Servicio Social contemporáneo. Trayectoria y desafíos». En La cuestión social y la formación profesional en Trabajo Social en el contexto de las nuevas relaciones de poder y la diversidad latinoamericana, editado por María Lorena Molina Molina, 17 50. Buenos Aires: Espacio.

Lukács, György. 2004. Ontología del ser social: El trabajo. Buenos Aires: Herramienta.

Maskrey, Andrew. 1989. El Manejo Popular de los Desastres. Estudio de Vulnerabilidad y Mitigación. Lima, Perú: ITD.

1993. «Vulnerabilidad y mitigación de desastres». En: Los Desastres no son Naturales, editado por Andrew Maskrey, 93-110. LA RED. Bogotá, Colombia: Tercer Mundo.

Molina, Molina, María Lorena. 2006. «El espacio local y los derechos económicos y sociales». En Política Social y Trabajo Social, editado por Nilsa Burgos Ortiz, 127148. Proyecto Atlantea. Puerto Rico: Universidad de Puerto Rico. 2007. «Cuestión Social y Trabajo Social: esbozo teórico histórico para interrogar la formación profesional contemporánea». Ponencia presentada en el IV Congreso Nacional de la AMETS Del proceso histórico a los desafíos y compromisos del Trabajo Social actual, Chihuahua, México, 7-9 de mayo.

Netto, José Paulo y Marcelo Braz. 2007. Economia política: uma introdução crítica. São Paulo, Brasil: Cortez.

Pereira Pereira, Potyara. 2002. Necesidades humanas. Para una crítica a los patrones mínimos de sobrevivencia. Brasil: Cortez.

UNISDR (Oficina de las Naciones Unidas para la Reducción del Riesgo de Desastres). 2015. Hacia el desarrollo sostenible: El futuro de la gestión del riesgo de desastres. Informe de Evaluación Global sobre la Reducción del Riesgo de Desastres. Ginebra, Suiza: UNISDR. 